\title{
LA ÓPTICA DE LEONARDO DA VINCI: LA MIRADA ARTÍSTICA COMO INTUICIÓN DEL PENSAMIENTO CIENTÍFICO
}

\author{
THE OPTICS OF LEONARDO DA VINCI: THE ARTISTIC LOOK AS INTUITION OF THE \\ SCIENTIFIC THOUGHT
}

SERGIO BARBERO BRIONES

Instituto de Óptica, CSIC

sergio.barbero@csic.es

RECIBIDO: $12 / 05 / 2016$

ACEPTADO: $17 / 07 / 2016$

Resumen: La mirada artística puede servir como estímulo de la intuición creadora de conocimiento científico. Este trabajo pretende desarrollar esta premisa filosófica a través del ejemplo histórico de Leonardo da Vinci. Su estudio científico sobre la óptica y su creación artística pictórica pueden parecer, en primera instancia, inextricablemente unidos. Sin embargo, en varias ocasiones sus hallazgos artísticos precedieron a su intuición científica sobre varios asuntos de óptica -sombras y penumbras, el azul del cielo, la pupila del ojo, etc.- lo cual respalda la reivindicación del arte como acicate para la creación científica.

Palabras clave: ciencia y arte, óptica, pintura, Leonardo da Vinci, intuición.

\begin{abstract}
The artistic look can serve as a stimulus to the creative intuition of scientific knowledge. This work aims at developing this philosophical premise through the historical example of Leonardo da Vinci. His scientific research in optics and his pictorial artistic creation may appear, at first glance, inextricable linked. However, on several occasions his artistic findings preceded his scientific intuition on various issues of optics -shadows and semi-darkness, the blue sky, the pupil of the eye, etc.- which supports the claim of art as an incentive for scientific creation.
\end{abstract}

Keywords: science and art, optics, painting, Leonardo da Vinci, intuition.

\section{La mirada artística y científica de la realidad}

La mirada sensible en el arte se deja impregnar por lo circundante, en un aparente movimiento de gracia sin metas aparentes. Ahora bien, el artista se acerca a la naturaleza con un doble talante: cándido pero a la vez metódico, de ahí que Thomas Mann afirme que el temperamento artístico es una "profunda e instintiva síntesis de disciplina y desenfreno" (Mann, 2002).

La mirada perspicaz de la ciencia a la realidad parte, como el arte, de la percepción sensorial, a través de la cual construye una imagen de aquella. Así arte y ciencia comparten el tratar - como mantenía Hermann von Helmholtz- no de transcribir la naturaleza sino de traducirla (Helmholtz, 1873, p. 135). Huelga decir que interesarse por lo que tienen en común ciencia y arte no significa 
confundirlas. Werner Heisenberg se preguntaba " ¿Qué diferencia hay entre un pintor abstracto y un físico teórico?", respondiendo, el mismo, que la diferencia se encontraba en el grado de fantasía utilizado a la hora de traducir la naturaleza. Mientras que el arte -al menos en su corriente abstracta- es considerado más excelso en tanto en cuanto más alto vuela la imaginación, contrariamente la ciencia se rige por un criterio de ajuste con lo observado.

A pesar de que ciencia y arte comportan una cierta mirada metafórica de la naturaleza, conviene explorar si se pueden beneficiar mutuamente, o si, en cambio, cualquier interferencia es más destructiva que constructiva. En esta relación, históricamente se ha dado más énfasis al influjo de las ideas científicas sobre los creadores de arte, aminorándose la influencia inversa. Sin embargo - como propone el historiador del arte Martin Kemp- no deberíamos ignorar la posibilidad: "de que algún elemento de naturaleza aparentemente científica pueda aparecer en las obras de un artista independientemente, o incluso por delante, de la ciencia correspondiente" (Kemp, 2000, p. 9).

Jorge Wagensberg sostiene que en la evolución humana surgió antes la percepción de la belleza - la sensibilidad estética- que de la inteligibilidad, prerrequisito del pensamiento científico (Wagensberg, 2004, p. 282). Y lo argumenta a través de un ejemplo. Existen pruebas de la fabricación, por parte del homo erectus, de piedras bifaces hace un millón de años. Estas nunca se llegaron a usar para fin utilitario alguno. Sin embargo poseían una fuerte simetría; siendo universalmente aceptado que la simetría se percibe como bello, la conclusión es que estas piedras bifaces se construyeron por el deleite estético. Por otro lado "la primera evidencia de conocimiento científico tiene unos treinta mil años y es un dibujo rupestre" (Wagensberg, 2004, p. 285). Por tanto, fue antes el arte que la ciencia. A pesar de que la verosimilitud de la tesis de Wagensberg pueda ser cuestionada, sí ayuda a subrayar la relevancia del conocimiento artístico en la historia de la humanidad.

La mirada artística puede servir como un primer acercamiento a la realidad. Los astrónomos exploran los cielos con un objetivo óptico con poco aumento pero con un gran campo visual, para, una vez localizada la estrella en el firmamento, cambiar a un objetivo con menor campo visual pero proporcionando mayor magnificación, con la finalidad de poder captar el detalle. De la misma manera la intuición artística puede servir como una primera exploración de la realidad que "localice" objetos de estudio, que ulteriormente se escudriñen mediante el método científico. Este primer discernimiento -en el sentido etimológico: cribar, separar-, posibilitado por la sensibilidad artística, es crucial ya que permite: "pasar desde la oscuridad de la ignorancia a distinguir el pensamiento por medio de los bellos oficios de la confusa, pero vívida, 
imaginería de los poetas" -Baumgarten, Aesthetica, 6-7, citado por (Kemp, 2000, p. 268). Aún, más rotundamente Ortega y Gasset: "en una de sus dimensiones la poesía es investigación y descubre hechos tan positivos como los habituales en la exploración científica"-citado por (Domingo-Moratalla, 2003).

\section{Leonardo da Vinci: fusión de fantasía e intelecto}

Uno de los testimonios clarificadores de la fructífera relación entre la sensibilidad artística y el conocimiento científico es la figura de Leonardo de Vinci. Para Luis Racionero, Leonardo "utiliza el arte para enseñar a mirar la naturaleza, provocando la investigación científica", mientras que hoy en día es "la ciencia quien motiva al arte" (Racionero, 2004, p. 22). Pero, ¿acaso la búsqueda de la belleza es ya una vía muerta en la cultura científica actual?

Leonardo era -en el buen y etimológico sentido de la palabra- un diletante de las ciencias y de las artes; su acercamiento al conocimiento brotaba del deleite ante la belleza de las manifestaciones sensoriales de la naturaleza. Su forma de desentrañar los misterios de ésta era buscando las conexiones que comparten un mismo patrón; el cual, a su entender, es siempre bello por estar sujeto a la armonía y la proporción. Leonardo: "enfatiza las íntimas conexiones entre la representación artística de las formas y el conocimiento intelectual de su naturaleza intrínseca y los principios subyacentes" (Capra, 2007, p. 27).

Da Vinci ejemplifica, en muchos sentidos, la nueva forma de ser y pensar del hombre moderno. Rechaza el magister dixit de la escolástica medieval y la mera especulación filosófica desligada de la experiencia sensorial, reivindicando la vecindad de lo cotidiano como objeto de estudio y la experiencia (sperienza) como principio ineludible para todo conocimiento. Así se expresa vehemente en los siguientes términos (da Vinci, 2010, p. 241):

Ahora piensa, ¡oh, lector!, qué confianza podemos tener en los antiguos que intentaron definir el alma y la vida -las cuales superan toda prueba- mientras que aquellas cosas que puedan ser conocidas con claridad en todo momento y probadas por la experiencia, permanecieron desconocidas durante muchos siglos o fueron entendidas erróneamente

Ciertamente, en ciertos momentos, Leonardo se posiciona en un empirismo extremo -"la experiencia nunca equivoca; es nuestra apreciación la que únicamente se equivoca, al esperar resultados no causados por los experimentos" 
(da Vinci, 2010, p. 244)- aunque es, tal vez, una mera reacción ante la desmesura de la escolástica medieval.

A pesar de este empirismo, la experiencia no es final sino inicio del saber que deviene con el intelecto humano. El fin de la experimentación es para Leonardo entender las razones del ser, las causas de la naturaleza. Si éstas fuesen comprendidas y sus efectos predichos, la experimentación dejaría de ser necesaria. La experimentación artística es un acercamiento espontáneo, no explícitamente dirigido - como la experimentación científica-, a esas causas de la naturaleza. Si bien no permite una comprensión plena, si esclarece vías de tránsito hacia el conocimiento.

Según una teoría del conocimiento medieval, los tres elementos primordiales en el intelecto son (Capra, 2007): el arte -que en sus días significaba la habilidad técnica-, la scientia, o sea el conocimiento, y la imaginatio o fantasia, es decir la imaginación creativa. Esto se materializa en una estructura morfológicamente tripartita y secuencial del cerebro (de ecos Galénicos): la primera zona es donde reside el sensus communis (receptor de los estímulos) en conjunción con la fantasia, la segunda zona contiene la zona racional del intelecto y la última es el repositorio de pensamiento, esto es la memoria. Leonardo introduce, en este esquema, un cambio aparentemente menor pero de una profunda hondura: desplaza la fantasía a la segunda cavidad, elevando el status de la imaginación (fantasia) a la de la razón (inteletto) (Kemp, 2006, p. 108). De esta manera el intelecto se nutre de la imaginación y viceversa. La figura 1 muestra el esquema fisiológico cerebral de Leonardo.

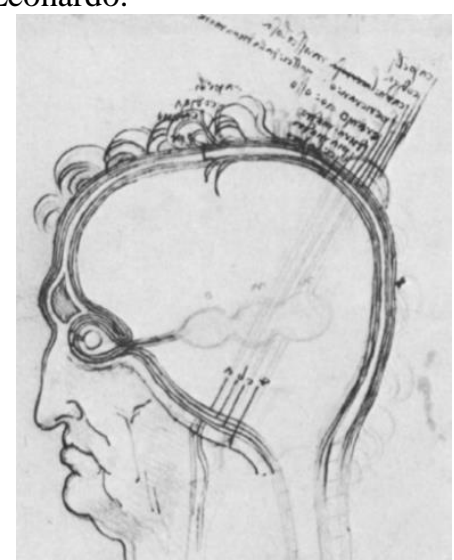

Figura 1. Las tres cavidades en el cerebro del ser humano (Manuscrito W, f. 12603r). 


\section{La óptica de Leonardo}

Siendo la experiencia sensorial el punto de partida de todo conocimiento, no todos los sentidos son igualmente valiosos. El de la visión ocupa un lugar predominante -siendo el oído "el segundo sentido en categoría" (da Vinci, 2010, p.143) - ya que las imágenes de la naturaleza es lo que mejor nos permite ahondar en ella. Su importancia es tal que "quien lo pierde se priva de la representación de todas las obras de la naturaleza" (da Vinci, 2010, p.53). De ahí que óptica y pintura, correlatos científicos y artísticos de la visión, cobren predominancia entre las inquietudes leonardescas. La pintura desempeña, en realidad, una cometido dual: "como arte y como herramienta de conocimiento científico" (Capra, 2007, p. 27).

En la Edad Media la ciencia de la óptica se denominaba perspectiva y se ocupaba del estudio geométrico de la representación visual de las escenas que observamos. La perspectiva, aplicada a la pintura, pretendía averiguar la mejor manera de generar una sensación visual de profundidad. Leonardo se enmarca plenamente dentro de esta revolución de la pintura que tiene lugar en el Quattrocento italiano - el pionero fue el florentino Filippo Brunelleschi (13771466) - con pintores como León Battista Alberti, Piero della Francesca, etc. Estos artistas eran conocedores de las teorías visuales de la Edad Media (Lindberg, 1976), herederas de los tratados griegos sobre óptica, y la reglas geométricas de la perspectiva lineal (proyección de escenas tridimensionales sobre superficies planas) que partían del concepto del cono visual desarrolladas en las susodichas teorías.

Ya que el dominio de la perspectiva permitía aumentar la sensación realista de las representaciones artísticas, no es de extrañar la importancia que Leonardo atribuyó a esta ciencia: "La perspectiva es el freno y timón de la pintura. La pintura se basa en la perspectiva, que no es otra cosa que un conocimiento perfecto de la función del ojo" (da Vinci, 2010, p. 61). Sin embargo, la innovación (inventio) pictórica no se basta sólo de este conocimiento de la perspectiva sino que necesita también de la fantasia.

Leonardo aprendió como aplicar los fundamentos de la perspectiva a la pintura a través de la versión italiana (1436) del libro De pictura de Alberti (Kemp, 2006, p. 10). También conocía la Perspectiva communis de John Pecham (Lindberg 1976) -libro, más bien básico, del que transcribió partes de su introducción en su Codex Atlanticus - y la Perspectiva de Witelo -sin duda el 
tratado de óptica más avanzado del occidente medieval ${ }^{1}$. Asimismo Francesca Fiorani sugiere que Leonardo tuvo acceso directo a la obra de Alhacen De aspectibus, a través de traducciones al italiano Degli aspetti (Fiorani, 2013, p. 286), el tratado que más influyó en el desarrollo de óptica moderna. Estos primeros estudios sistemáticos de textos científicos por parte de Leonardo coinciden con sus primeras anotaciones de observaciones de la naturaleza que datan de inicios de la década de 1480 .

Un periodo crucial es su estancia en la Universidad de Pavia (1490), donde entabló amistad con Fazio Cardano, profesor de matemáticas y experto en perspectiva, con quien mantuvo una fructífera relación. A partir de este momento comenzó la redacción de un nuevo cuaderno de notas, hoy en día conocido como Manuscrito C -Sobre luz y sombra, ms. 2174- y que terminó en 1491. El capítulo quinto de su Tratado de la pintura se basaría fundamentalmente en este manuscrito. En él trata de explicar la interacción de la luz con los objetos sólidos y la formación de sombras. En este periodo la máxima motivación es perfeccionar su pintura: la scientia sostén del arte.

Sin embargo la scientia per se acabó convirtiéndose en una imperiosa necesidad para el espíritu de Leonardo. La investigación sobre las sombras le condujo naturalmente al estudió de la visión. En 1492 comenzó un nuevo cuaderno de notas (Manuscrito A) en el que Leonardo parte del concepto, proveniente de la perspectiva clásica, de la pirámide visual. Si imaginamos un cono cuyo vértice se sitúa en el ojo y cuya base coincide con la superficie de un objeto, la teoría de la perspectiva nos dice -tercer postulado de la Óptica de Euclides- que sólo veremos aquello situado dentro de este cono. Este constructo matemático era universalmente aceptado, aunque la física de la visión era más controvertida. Unas teorías (intromisivas) sostenían que algo proveniente del exterior incide sobre el ojo, mientras que otras (extromisivas) argumentaban lo contrario, del ojo emanaba una sustancia que era la hacía posible la visión. A partir del Ms. A Leonardo (Lindberg, 1976, p. 161) fue defensor de las primeras $^{2}$, a pesar de sus coqueteos con las segundas en la década de 1480. Los objetos emiten réplicas de sí mismos (similitude) que se propagan por el aire siguiendo trayectorias rectilíneas. Estas similitude se pueden captar si se propagan a lo largo de rayos -estos no interfieren mutuamente, a pesar de que se crucen- que se encuentren contenidos dentro del cono visual.

Casi dos décadas después Leonardo retomaría sus estudios en óptica -entre 1505 y 1510 (Heitz, 2009)- revisando sus viajes ideas y dándolas forma en un

\footnotetext{
1 "Buscar Witelo en la biblioteca en Pavia" (Codex Atlanticus, folio 225r). Leonardo aprendió latín de manera autodidacta a partir de 1494 (Clark, 1988, p. 51) pero siempre tuvo graves carencias.

${ }^{2}$ Leonardo conocía ya la teoría intromisiva en la década de 1470 (Fiorani, 2013, p. 270).
} 
nuevo cuaderno de notas: Manuscrito D. Asiduamente denominado Sobre el ojo, este manuscrito es un compendio sucinto (diez folios) pero sumamente relevante para entender las últimas ideas de Leonardo sobre oftalmología y óptica. Otro manuscrito (F) también contendría cometarios sobre fenómenos ópticos. Por último, al final de su vida, se interesaría sobre los fenómenos luminosos del espacio, recopilando sus pensamientos al respecto en el Codex Leicester (Fiorani, 2013 , p. 24). De alguna manera -como sugiere Kemp (Kemp, 1977)- se puede decir que la evolución de las ideas sobre óptica de Leonardo se puede asimilar como "paralelo, en un microcosmos, al desarrollo histórico desde Euclides a Alhacén y más allá".

En un balance global ¿qué se puede decir sobre las contribuciones científicas de Leonardo a la óptica? Más allá de la controversia de si éstas fueron o no originales -asunto en cierto modo irrelevante para la historia de la óptica ya que sus manuscritos no fueron estudiados sistemáticamente hasta finales del siglo XVIII-, lo que nos interesante aquí es ver cómo su intuición artística (fantasia) fue el catalizador de algunos de sus más relevantes descubrimientos (scientia) de óptica. En cierto modo, se trata de invertir el propio método de Leonardo, con el que pretendía ir de la scientia al arte. Lo veremos en una serie de ejemplos paradigmáticos.

\section{Sombras y penumbras}

El marco teórico de la perspectiva medieval -Perspectiva communis de John Peckham y De perspectiva de Witelo- establecía que en el espacio detrás de un objeto iluminado, o bien había luz, o bien había ausencia de ella, en cuyo caso surgía una sombra homogénea (Smith, 2015, p. 310). Sin embargo, en su atenta mirada de pintor, Leonardo, cómo probablemente otros pintores igual que él, se percató de que estas sombras contienen matices; distan de ser perfectamente uniformes y delimitadas por bordes abruptos, más bien eran en "sí mismas de distintos grados de oscuridad" (Codex Atlanticus folio 250r, Da Vinci, 2004, p. 164). De hecho, esta observación, es en buena parte el fundamento de la técnica pictórica del claroscuro (chiaroscuro).

Clark (Clark, 1988, p. 63) sostiene que Leonardo alcanzó un elevadísimo grado de perfección en el uso del claroscuro en torno a 1489, en unos dibujos sobre cráneos (figura 2) que se conservan en la Biblioteca Real del Castillo de Windsor; precisamente un año antes de sus elaborados análisis geométricos de la luz sobre objetos geométricos, tales como esferas o cilindros, que conformarían su ms. C. Es, pues, razonable deducir que una vez que Leonardo había 
"extenuado" parte de las posibilidades artísticas que su atenta observación de la naturaleza le proporcionaron, se vio interpelado a profundizar en sus hallazgos artísticos a través del conocimiento científico. La observación de los ricos matices de luces y sombras presentes en la naturaleza le condujo a realizar una serie de experimentos sistemáticos sobre problemas de iluminación.
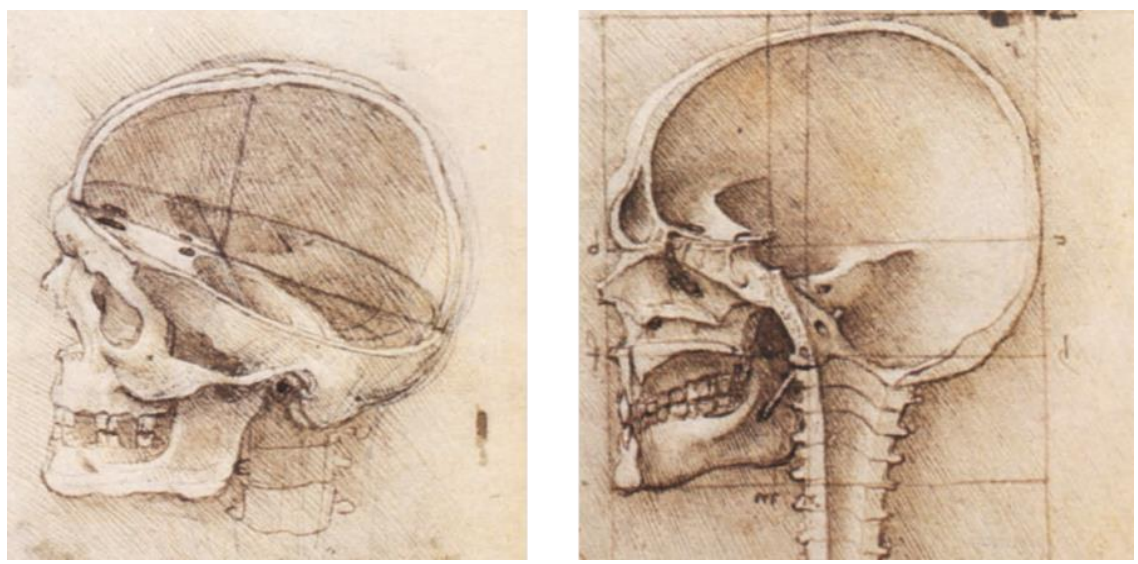

Figura 2. Dibujos de cráneos de Leonardo (Biblioteca Real Castillo de Windsor folio 19057r)

Lo nuclear de las ideas leonardescas sobre la formación de sombras graduales se puede condensar en un esquema que aparece en el ms. C. La parte derecha de la figura 3 es un esquema simplificado pero que ayuda a entender lo esencial de la deducción geométrica de Leonardo. Un objeto esférico bloquea la luz proveniente de un objeto emisor en forma de línea horizontal. Concentrémonos únicamente en tres fuentes puntuales pertenecientes a esta línea $(\mathbf{a}, \mathbf{b} \& \mathbf{c})$, de las cuales emanan tres conos visuales. La base del cono proveniente de a viene determinada por el segmento entre a' y a'". Análogamente de b tendremos el segmento b'- b'" y de c: c'-c'. Si superponemos la intensidad luminosa de los tres conos podemos deducir tres zonas diferenciadas: 1) la región comprendida entre la esfera y el segmento a'-c', en la cual no llega luz de ninguno de los tres conos, por lo que habrá oscuridad total, lo que Leonardo denominó ombra semplice; 2) la delimitada por los conos f-b'-a' y g-c'-b", donde únicamente llega luz del cono visual proveniente de $\mathbf{b}$, de lo cual se deduce que la sombra será sombra parcial, digamos de intensidad I; 3) la delimitada por los conos f-c'b' y g-b"'-a", donde llega luz de dos conos visuales con lo cual la intensidad de 
luz será el doble que en el caso anterior: 2I. De aquí que la sombra en esta última región poseerá un grado de oscuridad que es la mitad de la existente en la región 2. Las sombras de las regiones 2 y 3 son parciales, comparadas con la región 1 , debido a la superposición de varios conos visuales, de ahí que Leonardo las denominase ombra composta ${ }^{3}$.

Si se aumenta el número de conos visuales el análisis se vuelve más complejo pero esencialmente seguiremos teniendo una superposición variable de conos, lo cual conlleva ineludiblemente una gradación en la cantidad de luz, y por tanto en el tipo de sombra.

Leonardo también clasificaba las sombras siguiendo otro criterio: si son directamente generadas por un objeto que bloquea la luz se denominaban ombra originale o primitiva, mientras si son percibidas en la superficie de un objeto pero generadas fuera de éste se llamaban ombra dirivativa. Lo que en la terminología de arte actual se denominan sombras propias y sombras proyectadas.
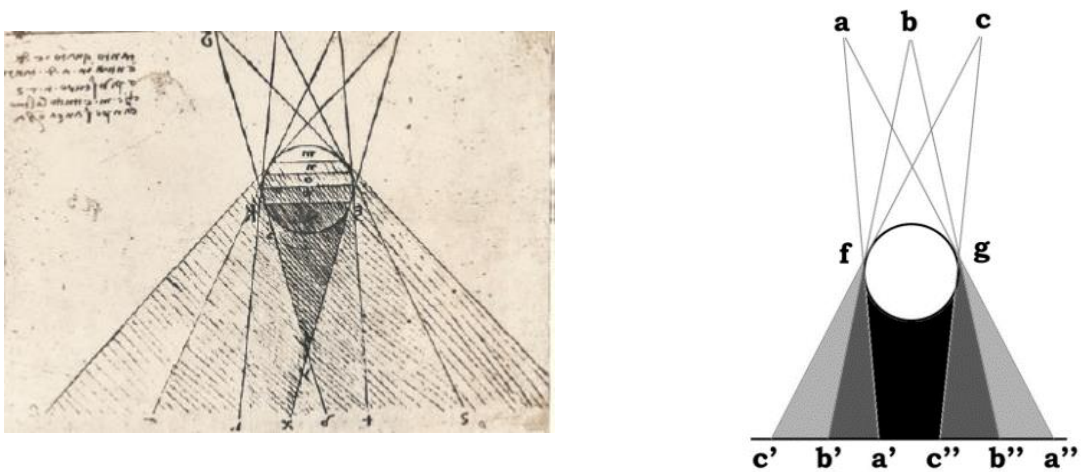

Figura 3. Izquierda: Esquema de Leonardo para representar sombras y penumbras generadas por una esfera. Derecha: Adaptación conceptual de su figura.

El estudio de Leonardo sobre las reflexiones de la luz no solo versaba sobre la gradación de la escala de grises presentes en las sombras sino también acerca del color de las sombras. Éste se produce porque a la zona de sombra llegan conos de luz provenientes de la reflexión de objetos próximos de distinto color: "la sombra participa siempre del color del cuerpo que la engendra" (Biblioteca Real

\footnotetext{
${ }^{3}$ Johannes Kepler acuñaría el término penumbra (del latín paene 'casi' y umbra) para esta sombra parcial.
} 
Castillo de Windsor folio 19076r, Da Vinci, 2004, p. 243). Los rayos visuales transportan, pues, información no sólo de la forma de los objetos que los engendran sino también de su color. Ahora bien, a veces se producen situaciones en las que por el relieve de un cuerpo y la localización de la fuente luminosa, algunas partes de aquel sólo las podamos ver por la iluminación indirecta reflejada en otra parte del cuerpo. En este caso, veremos aquella parte del cuerpo del color de ésta. Leonardo ilustró esta idea con un dibujo, adaptado en la parte derecha de la figura 4.

Si a es la luz y b está iluminado por la línea de esa luz, c, que la luz no puede ver, ve tan solo la parte iluminada que, supongámoslo así es roja. En tal caso, la luz reflejada por ella se asemejará a la superficie que la causa y teñirá de rojo la cara c." (Codex Ashburnham I folio 32v, Da Vinci, 2004, p. 252).

Este efecto se ve maravillosamente ilustrado en el cuadro de Leonardo: La belle ferronière (c. 1490-1495). Un delicado reflejo rojizo (equivalente al punto c del esquema de Leonardo) se vislumbra en la parte inferior de la mejilla izquierda, proveniente del reflejo de la luz sobre el vestido de color rojo parduzco (punto b).

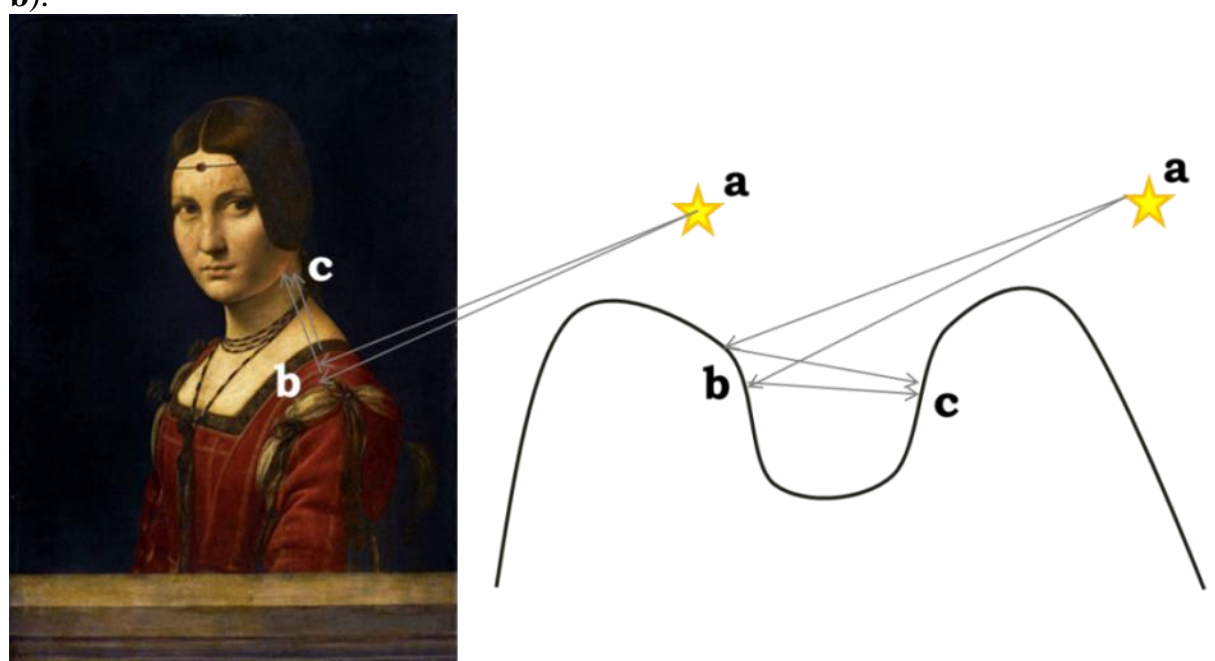

Figura 4. La belle ferronière y figura adaptada de la que aparece en $M s$. Ashburnham I folio 32v. 
Estas reverberaciones luminosas de los colores sería desde entonces un recurso pictórico recurrente en diversas tendencias artísticas, Leonardo: "deja entrever también en estas notas el principio impresionista de que todas las cosas se reflejan más o menos en otras y que, además, no existen las sombras negras" (Clark, 1988, p. 114).

\section{El azul del cielo y la perspectiva aérea}

Como ya se mencionó antes, la finalidad del uso de la perspectiva en pintura era crear una sensación de profundidad cuando contemplamos una representación en la superficie plana de un lienzo, según queda explícito en el Tratado de la pintura (Fiorani, 2013, p. 85). Ahora bien, a parte de la perspectiva lineal, desde sus primeros tiempos milaneses, Leonardo identificó otros dos tipos de perspectiva. La prospettiva de spedizione -basada en la observación de que lo situado en la lontananza se ve con menos resolución espacial que lo próximoque conlleva la difuminación de las formas y colores con la distancia y la prospettiva aerea (o dei colori) que apela a nuestra experiencia sensorial previa: objetos lejanos observados a través de la atmósfera adquieren un tono azulado, por lo que en pintura se crea una sensación visual de lejanía cuando se utilizan tonos azulados y difuminados.

Como recurso pictórico, la perspectiva aérea era conocida y utilizada en la Antigüedad greco-latina. Ptolomeo lo menciona en su tratado de óptica (escrito entre el 160 y 170 d.C.) "la localización de objetos más brillantes, tales como la luna o el sol, se perciben como más cercanos, por lo que los pintores de murales usan colores débiles tenues para representar cosas que quieren que muestren cierta distancia" (Ptolemy, 1996, p. 120). Sin embargo, da Vinci lo redescubre para el Renacimiento, acuñando el término prospettiva aérea y enfatizando el color azulado que adquiere la lontananza: "en un aire de uniforme densidad, las cosas últimas vistas a través de él, como las montañas, parecen, por culpa de la gran cantidad de aire interpuesto entre tu ojo y la montaña, azules" (Codex Ashburnham I folio 25r, Vinci, 2004, p. 263). Influido por los patrones geométricos de la perspectiva lineal, Leonardo pretendió descubrir una regla matemática válida para la perspectiva aérea, la cual establecía que el color disminuye de manera regular en razón de cuatro quintos de su valor previo por cada veinte brazos de distancia recorrida (Codex Ashburnham II folio 22v, Kemp, 2006, p. 116).

Da Vinci hizo un uso profuso de la perspectiva aérea en su pintura, en cuadros como: Retrato de Ginevra Benci (1478-1480), Madona Litta -c. 1490, 
aunque de adscripción controvertida a Leonardo- la segunda versión de $L a$ virgen de las rocas (1493-1495), Estudio de ropaje para Santa Ana, la Virgen y el Niño (c. 1507 o 1517), o La Gioconda (1503-1506). La figura 5 muestra como el fondo azulado y difuminado del paisaje realza el volumen del rostro de la Gioconda.

Leonardo retorna a Milán en el verano de 1508 donde vuelve a trabajar para la corte en múltiples ocupaciones al servicio de Charles d'Amboise. En esta nueva etapa milanesa Leonardo se vuelca aún más en sus observaciones de la naturaleza, quizá motivado por la cercanía de los Alpes. De esta época data el Manuscrito G (c. 1510-1511 y 1515) y el Codex Leicester (c. 1506-1509), donde anota comentarios sobre diversos efectos luminosos y analiza en detalle la perspectiva aérea.

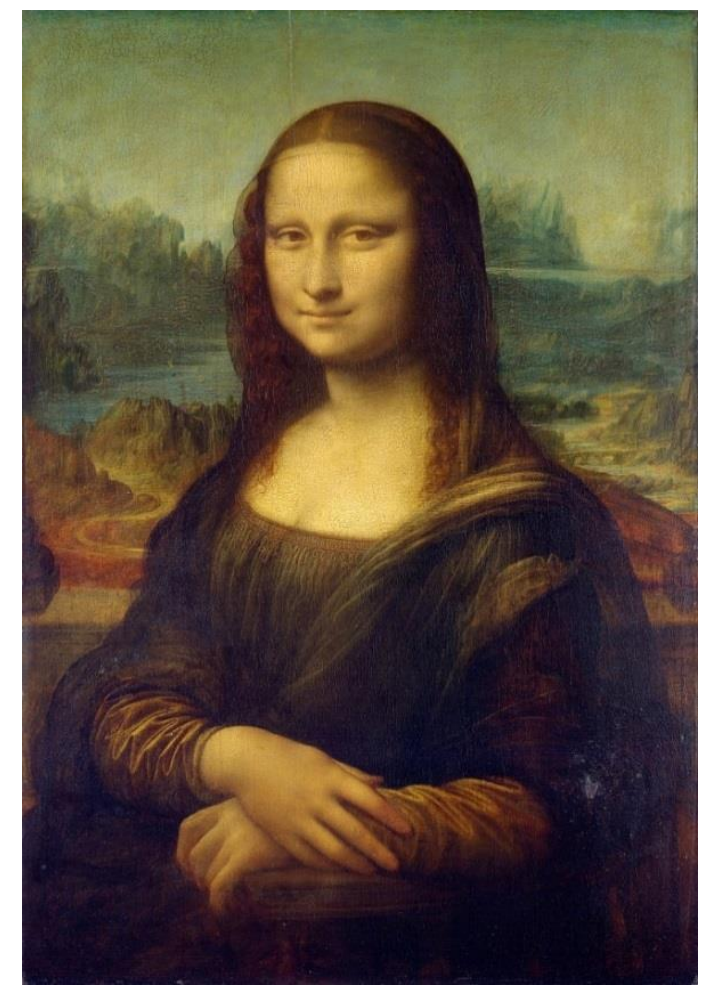

Figura 5. Perspectiva aérea en el cuadro Mona Lisa. 
En esta época la observación empírica de la naturaleza, espoleada por su sensibilidad artística, muta en una nueva intuición científica en la mente de Leonardo que trata de dar respuesta a la causa física de la perspectiva aérea: ¿por qué los objetos vistos a través de la atmósfera se ven azules? La inspiración le vino cuando escaló un pico de los Alpes (Codex Leicester folio 4r., Biblioteca Leonardiana, 2016)

Dico, l'azzurro, (che) in che si mostra l'aria, non essere suo propio colore, ma è causato da umidità calda, vaporata in minutissimi e insensibili attimi, la quale piglia dopo sè la percussion de'razzi Solari e fassi luminosa sotto (le ten) la oscurità della immense tenebre della regione del fuoco, che di sopra le fa coperchio; e questo vedrà, come vid'io, che andrà sopra mom Boso, giogo dell'Alpi...

El color azul de la atmosfera, intuye 'acertadamente' Leonardo, "no es su propio color" sino que se debe a la interacción de los rayos solares con la humedad circundante: "presente en diminutos e imperceptibles átomos" ${ }^{4}$. Posteriormente, Leonardo incide en la humedad como factor causante del color azul, demostrándolo no ya con una mera observación sino con algo que bien podría ser un 'experimento': "la diferencia puede verse en los átomos de polvo y en los átomos de humo de los rayos solares que, a través de un orificio en la pared, penetran en un lugar oscuro: un rayo parece ceniciento y el otro, de sutil humo, parecerá de un azul bellísimo" (Codex Leicester folio 4r, Da Vinci, 2004, p. 267).

Por tanto, el cielo no es azul sino que lo parece por la interacción de la luz con la atmósfera: respuesta válida para uno de los grandes interrogantes de la historia de la ciencia óptica y que resuena como la inocente pregunta de un niño movido por su innata curiosidad ¿por qué el cielo es azul?

\section{La pupila del ojo y el esfumado}

Una de las aportaciones de Leonardo a la óptica fisiológica fue la observación y estudio de la miosis pupilar. Esto es, la capacidad de la pupila de cambiar su tamaño. Conviene matizar que la palabra pupila (popilla) es para Leonardo polisémica (Heitz, 2009), en ciertas ocasiones denota la córnea, en otras la cámara anterior del ojo aunque las más de las veces tiene el mismo significado

\footnotetext{
${ }^{4}$ La dispersión de Rayleigh por parte de las moléculas que componen el aire es la principal explicación científica actual de por qué el cielo es azul.
} 
que en la actualidad: la apertura que fija la cantidad de luz que entra en el ojo. Es el significado que utilizaremos aquí.

Leonardo dedujo que el tamaño de la pupila cambiaba esencialmente debido a la mayor o menor luminosidad del objeto - por tanto con la finalidad de capturar más o menos luz.

Questa nostra popila cresce e diminuisce secondo la chiarità o scurità del suo obietto, e perché con qualche tempo fa esso crescere e discrescere, esso non vede cosi presto uscendo [d]al lume andando all'oscuro, e similmente dallo scuro al luminoso. E questa cosa già m'ingannò nel dipignere un occhio e di lì la 'mparai" (Codex Foster II folio 158v, Biblioteca Leonardiana, 2016)

Lo relevante, para este estudio, de este descubrimiento es que -como el propio Leonardo confiesa- la inspiración le vino durante su práctica artística; tras una falsa apariencia sensorial (ingannò) cuando intentaba pintar un ojo en torno a 1490 (Keele, 1983 p. 73, Capra, 2007, p. 239, Da Vinci, 2004, p. 105). Varios autores consideran que la miosis pupilar fue el descubrimiento más original de Leonardo en óptica fisiológica (Lindberg, 1976 p. 163, p. 1983, Keele, 1983, p. 71); por lo que el hecho que éste surgiese mientras pintaba un cuadro pone de relieve como la sensibilidad artística alimentaba el genio creativo científico de Leonardo.

La función de la pupila del ojo como reguladora de intensidad luminosa permitió a Leonardo posicionarse en cuanto a la antigua controversia entre la teoría intromisiva y extromisiva del ojo. Para ello uso argumentos similares a los previamente utilizados por Alhacén ${ }^{5}: 1$ ) el efecto perjudicial de un exceso de luz repentino "l'occhio uso nelle tenebre che subito veda la luce, riceve detrimento" (Ms. C folio 16r, Biblioteca Leonardiana, 2016); 2) el fenómeno de postimágenes: "Después de la visión permanecen aún en el ojo las imágenes del objeto aprehendido" (Codex Atlanticus 203r, Da Vinci, 2004, p. 106). Leonardo llego incluso a pretender describir el mecanismo fisiológico que permitía la contracción pupilar.

Las intuiciones leonardescas no fueron siempre correctas ${ }^{6}$. Leonardo asignó una funcionalidad adicional a la pupila: "maggiore popilla vede maggiore obbietto" (Ms. D folio 7r, Biblioteca Leonardiana, 2016), el tamaño de la pupila no sólo determina la cantidad de luz sino también la percepción del tamaño de los objetos, apreciación incorrecta. La desacertada intuición vino falsamente avalada por una deducción geométrica errónea ya que partía de nociones de

\footnotetext{
${ }^{5}$ Ver discusión en (Keele, 1983, p. 71).

${ }^{6}$ Conviene señalarlo para desmitificar su tan loada genialidad.
} 
óptica fisiológica inexactas (Ms D, folio 7r, Biblioteca Leonardiana, 2016). En cualquier caso, prueba una vez más como el artista-científico trataba continuamente de comprobar sus ideas de fantasia a través de su intelleto.

Una de las diferencias fundamentales en las nociones ópticas de Leonardo, de su época de madurez respecto de su primera etapa milanesa, fue precisamente la relevancia asignada a la función de la pupila. La captura de las réplicas de los objetos (similitude) no se produce en único punto sino en toda la superficie pupilar. Ahora bien la potencia visual (virtù visiva), es decir, la capacidad de producir sensación visual, es mayor para los rayos que pasan por el centro de la pupila. Los rayos luminosos son enviados desde la "imprensiva al sensus communis donde son valorados" (Ms. D, folio 2v, Biblioteca Leonardiana, 2016), siendo el sensus communis la facultad del alma que permite la percepción visual -de nuevo resuenan las ideas de Alhacén.

La característica no puntual de la virtù visiva conlleva algunas implicaciones especialmente relevantes para entender ciertas experiencias visuales. Una de ellas es la percepción visual de contornos o bordes. Leonardo afirma taxativamente: "L'occhio non sarà mai capace del vero termine che han le figure di qualunche corpo campeggianti in loco remoto" (Ms. D folio 10v, Biblioteca Leonardiana, 2016) La presencia de un fondo impide ver con claridad el contorno de cualquier cuerpo (termine che han le figure). Leonardo deduce esto siguiendo la ilustración inferior del Ms. D folio 10v, que hemos adaptado en la Figura 6.

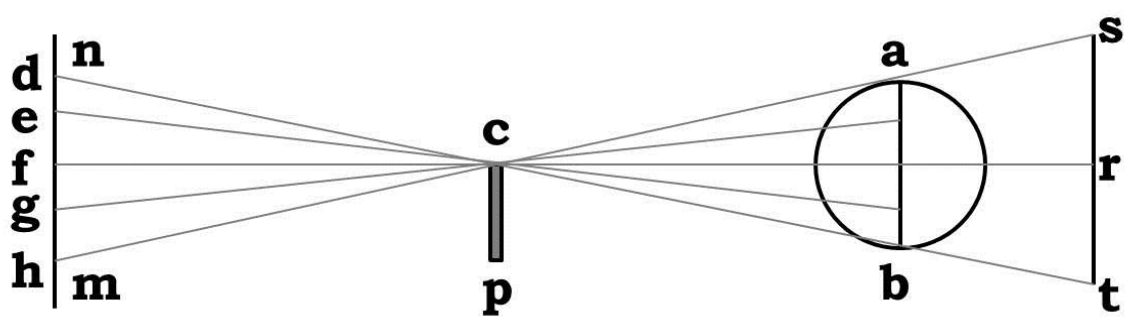

Figura 6. Representación de la perspectiva de contornos (adaptado de ilustración Ms. D folio 10v)

La figura 6 representa un objeto $c p$ con un transfondo $n m$ y un ojo que lo observa cuya pupila es $a b$. Si tratamos de fijar nuestra atención en el borde $c$ del objeto $c p$ no podremos evitar que lleguen al ojo rayos provenientes del fondo $\mathrm{nm}$. Del extremo inferior $h$ un rayo incidirá en el borde superior de la pupila $a$, y, viceversa, por el punto más inferior de la pupila $b$ pasará un rayo proveniente del 
punto más superior del fondo $d$. Esto provoca que $c$ no se vea como un único punto $r$ sino como una mancha $s t^{7}$. Es fácilmente deducible, por un simple argumento geométrico, que el tamaño de la mancha aumentará según el borde se acerque al ojo. Conviene matizar que, en cierto sentido, esto presenta cierta contradicción con la prospettiva spedizione, que predice que las cosas lejanas se vean borrosas por un pérdida de agudeza visual: "de lejos todos los contornos resultan indiscernibles" (Codex Ashburnham 10r, Da Vinci, 2004, p. 262).

Ahora bien, ¿fue este razonamiento deductivo el seguido por Leonardo para descubrir la visión borrosa de contornos? Un cauto Kemp mantiene que no podemos saberlo (Kemp, 1977): "Es imposible decir si la existencia del efecto del emborronamiento de borde fue deducido por Leonardo en base a la premisa de que la virtù visiva se encuentra dispersada a lo largo de toda la pupila, o si más bien fue primero observado y luego explicado". Nuestra hipótesis es, sin embargo, que Leonardo siguió el camino inverso a la deducción: la inducción.

La observación de que los bordes aparecen emborronados en la presencia de un fondo es una experiencia que no pudo pasar desapercibida a un observador tan sagaz como Leonardo. Esta observación, en conjunción con la prospettiva spedizione, justifican la funcionalidad figurativa del sfumato; técnica pictórica profusamente usada por Leonardo, con quien alcanzó sus máximas cotas artísticas.

El sfumato, como técnica pictórica de la pintura al óleo, fue inventado por los primitivos flamencos. Consiste en la aplicación sucesiva de veladuras (finas capas de pintura con cierto grado de traslucidez) sobre el lienzo con una primera base más o menos oscura. Las veladuras crean, por reflexión difusa en las distintas capas $^{8}$ una superposición de delicadas interferencias luminosas que crean una sensación de apariencia difuminada, la cual se manifiesta principalmente en los contornos. El logro visual, entre otros, es proporcionar corporeidad, plasticidad a las figuras del cuadro. Así, como acertadamente afirma Janis Bell (Bell, 2008), el sfumato es tanto técnica como percepción.

El sfumato está presente en las primeras obras de Leonardo 9 , aunque no consigue un dominio claro de la técnica hasta La virgen de las rocas (14831485). Leonardo lo utilizaba esencialmente para imitar lo percibido por la visión

\footnotetext{
${ }^{7}$ En términos modernos, la imagen de los contornos esta emborronada porque la imagen formada por objetos situado a varios planos se encuentra desenfocada, dejando aparte los fenómenos de difracción óptica de bordes.

${ }^{8}$ Técnicamente este fenómeno se denomina scattering incoherente (Simonot, 2004)

${ }^{9} \mathrm{Su}$ hagiógrafo Giorgio Vasari afirma que incluso aparece ya en las primeras manifestaciones relevantes de Leonardo como aprendiz en el taller de Verrocchio: el ángel del Bautismo de Cristo (1475-1478) (Fiorani, 2013, p. 88).
} 
creando sensación de relieve y poder representar los bellos atributos de la naturaleza: grazia y dolcezza (Bell, 2008). Aunque tampoco se debe ignorar una funcionalidad simbólica: los contornos difuminados de la virgen del susodicho cuadro le sirvieron para simbolizar la inmaculada concepción como emergiendo de las sombras del pecado (Olszewski, 2011).

El potencial de la técnica del esfumado aparece en el último gran cuadro de Leonardo: San Juan Bautista, elaborado entre 1513 y 1516. Scientia y ars se retroalimentan, el conocimiento del efecto de emborronamiento de bordes seguramente espoleó el espíritu creativo de Leonardo en este último cuadro. El San Juan Bautista aúna de manera prodigiosa las técnicas de sfumato y chiaroscuro para recrear percepción de volumen; en efecto, el semblante del santo, sobre un fondo completamente oscuro y con unos contornos difuminados, parece, para quien lo contemple de frente, emerger del lienzo.

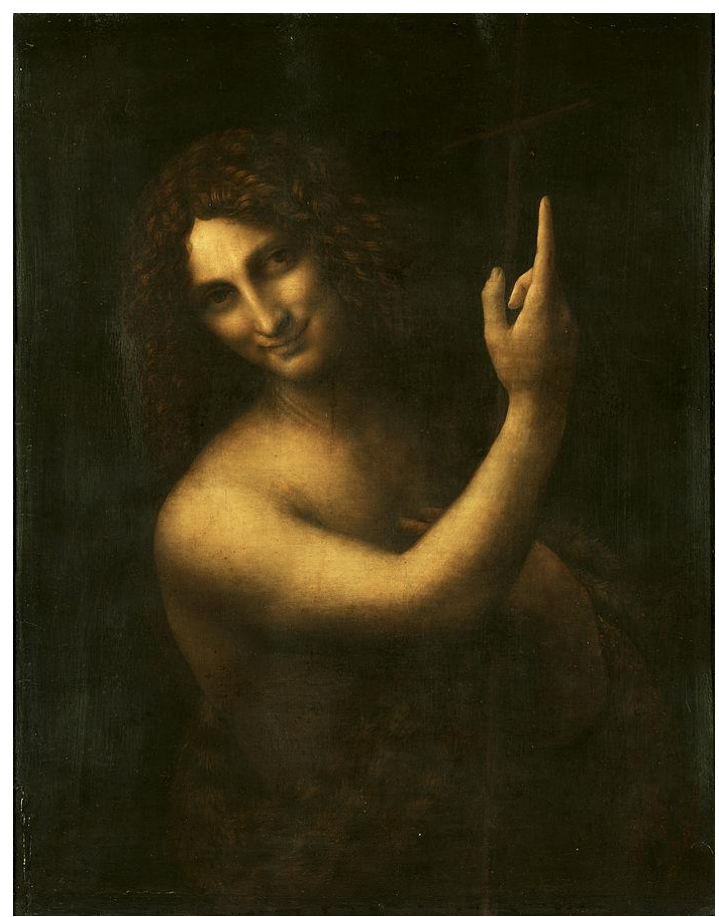

Figura 7. Técnica de sfumato en San Juan Bautista. 


\section{La luz de la Luna cenicienta}

Leonardo abordó la naturaleza como un sistema integral; su método de investigación era buscar los isomorfismos, las estructuras comunes en todas las manifestaciones de la naturaleza. Así, Leonardo, se acercó a la astronomía con el andamiaje de la óptica; el objetivo era estudiar la apariencia visual de los astros y como la luz se transmite de unos astros a otros. La luna fue una de las grandes fascinaciones de Leonardo. Sostenía - al contrario que la extendida opinión, avalada por Aristóteles- que la luna no emitía su propia luz, sino que ésta proviene de la reflejada por el sol: "La luna non ha lume da sé, se non quanto ne vede il sole, tanto l'alumina" (Codex Arundel folio 94v, Biblioteca Leonardiana, 2016).

Un fenómeno más sutil es el llamado la luz cenicienta de la luna. Es la débil iluminación que recibimos de la luna proveniente de la zona no directamente iluminada por el sol. El origen de esta luz fue una larga controversia en la historia de la astronomía. Otra de las extraordinarias audacias de Leonardo fue la de sugerir que ésta débil luz provenía de la luz reflejada por el sol en la tierra. La luz cenicienta no sería otra cosa sino el segundo reflejo de la luz solar en la luna tras un primer reflejo en la tierra. Ahora bien, como buen conocedor de los patrones de iluminación generados por una esfera, Leonardo concluyó que la superficie lunar no podía ser simplemente una superficie esférica perfecta: "Esto no podría ocurrir si la esfera de agua, que en gran parte cubre la luna, fuese uniformemente esférica, ya que en tal caso habría una imagen del sol para cada ojo" (Codex Arundel 94v, Da Vinci, 2008, p. 51). Achacó lo patrones irregulares de los reflejos a la presencia de una superficie de agua irregular en la superficie lunar.

Su pasión por la luna y por querer desentrañar lo que en ella existe, nos lleva a una última y maravillosa predicción tecnológica de Leonardo, la de "construir lentes para ver la luna magnificada" (da Vinci, 2008, p. 351); esto, es un telescopio, un siglo antes de que Galileo viese la superficie lunar con uno diseñado por él mismo. Dada la ausencia de pruebas documentales, no se puede avalar la sospecha del historiador Lucio Russo, quien mantenía que la intuición de Leonardo sobre el telescopio sólo pudiese surgir tras la lectura de ese conocimiento en fuentes de origen griego (Russo, 2004, p. 343). Aunque, de la misma manera, el supuesto antagónico, Leonardo como inventor del telescopio, propuesto por Domenico Argentieri en 1939, es igualmente inasumible (Fiorani, 2013, p. 21). Si hemos cumplido con el objetivo de este ensayo, esperamos, en cambio, que ahora sea más plausible pensar que fue el espíritu inventivo de 
Leonardo, espoleado por su sensibilidad artística, lo que le condujo a tal profética predicción.

Bien quisiéramos que este breve recorrido por las lúcidas intuiciones leonardescas sobre la óptica sirva de acicate para reivindicar la idea Aristotélica de que la intuición es una de las principales fuentes de conocimiento, ya que ésta no es otra cosa sino la capacidad de discernir lo universal en lo particular. La etimología de la palabra es reveladora, compuesta de 'in' (hacía dentro) y 'tueri' (observar, mirar), denota, pues, mirar algo con profundidad, con suficiente claridad y discernimiento. La intuición artística (fantassia) puede, y debe, ser aprovechada para transitar hacia nuevos saberes no sólo artísticos sino también científicos.

\section{Referencias}

Bell, J. Sfumato and acuity perspectiva, in Leonardo da Vinci and the ethics of style. Manchester, Manchester University Press, 2008.

Biblioteca Leonardiana, e-Leo History of science and technological digital archive [en línea]. [Fecha de consulta: 22 de marzo de 2016]. Disponible en: http://www.leonardodigitale.com/

Capra, F. The Science of Leonardo: Inside the Mind of the Great Genius of the Renaissance. Knopf Doubleday, 2007.

Clark, K. Leonardo da Vinci. Madrid: Alianza Editorial, 1988.

Da Vinci, L. Tratado de la pintura. Edición preparada por González García, A. Madrid: Ediciones Akal, 2004.

Da Vinci, L. Leonardo da Vinci Notebooks. Selected by Richter, I. Oxford: Oxford University Press, 2008.

Da Vinci, L. Cuaderno de notas. Leonardo da Vinci. Madrid: Edimat Libros, 2010.

Domingo-Moratalla, T. "La hermenéutica de la metáfora: de Ortega a Ricoeur." Espéculo. Revista de estudios literarios. 24(2003) pp. 1139-3637.

Fiorani, F., Nova, A. editores Leonardo da Vinci and optics. Venica: Marsilio Editori, 2013.

Heitz, R.F. "À propos du Manuscript D "Dell'occhio" de Léonard de Vinci". Historie des sciences médicales, 18-2(2009), pp. 199-208.

Helmholtz, H. von. "On relation of optics to painting", in Popular lectures on scientific subjects. London, Thoemmes Press, 1873.

Kemp, M. Leonardo and the visual pyramid. Journal of the Warburg and Courtauld Institutes, 40(1977) pp. 128-149. 
Keele, K.D. Leonardo da Vinci's elements of the science of man. New York: Academic Press, 1983.

Kemp, M. La ciencia del arte. La óptica en el arte occidental de Brunelleschi a Seurat. Madrid: Akal, 2000.

Kemp, M. Leonardo da Vinci: The marvellous works of nature and man. New York: Oxford University Press, 2006.

Kepler, J. Optics. Paralipomena to Witelo \& Optical part of astronomy. Translated by Donahue, A. H. Santa Fe: Green Lion Press, 2000.

Lindberg, D. C. Theories of vision from al-Kindi to Kepler. Chicago: University of Chicago Press, 1976.

Mann, T. Muerte en Venecia. Madrid: El País, Clásicos del siglo XX, 2002.

Olszewski, E.J. "How Leonardo invented sfumato". Notes in the history of art. 31 (2011), pp. 4-9.

Ptolemy, Ptolemy's theory of visual perception: an English translation of the Optics. Translated by A. M. Smith. Philadelphia: American Philosophical Society, 1996.

Racionero, L. Leonardo da Vinci. L'Hospitalet: Ediciones Folio, 2004.

Russo, L. The forgotten revolution: how science was born in 300 BC and why it had to be reborn. Berlin: Springer, 2004.

Simonot, L, Elias, M. \& Charron, E. Special visual effect of art glazes explained by the radiative transfer equation. Applied Optics, 43(2004), pp. 2580-2587.

Smith, A.M. From sight to light. The passage from ancient to modern optics. Chicago: The University of Chichago Press, 2015.

Wagensberg, J. La Rebelión de las formas, o, Cómo perseverar cuando la incertidumbre aprieta. Barcelona: Tusquets, 2004.

\section{Procedencia de las figuras}

Figura 1: Figura extraída de reproducciones de Manuscrito W, f. 12603r. Dominio público.

Figura 2: Figura extraída de reproducciones de Biblioteca Real Castillo de Windsor folio 19057r. Dominio público

Figura 3: Cuadro La belle ferronière. Dominio público. Tomada de https://es.wikipedia.org/wiki/La_Belle_Ferroni\%C3\%A8re\#/media/File:La_Bell e_Ferronni\%C3\%A8re.jpg

Figura 4: Figura de elaboración propia a partir de figura (dominio público) extraída de reproducciones de Manuscrito C de Leonardo. 
Figura 5: Cuadro Mona Lisa. Dominio público. Tomada de https://es.wikipedia.org/wiki/La_Gioconda\#/media/File:Mona_Lisa,_by_Leonar do_da_Vinci,_from_C2RMF_retouched.jpg

Figura 6: Figura de elaboración propia.

Figura 7: Cuadro San Juan Bautista. Dominio público. Tomada de https://es.wikipedia.org/wiki/San_Juan_Bautista_(Leonardo) 
